\title{
LED Applications in Railway Signals: Wavelength and Intensity vs Temperature Variation
}

\author{
Giuseppe Schirripa Spagnolo, Donato Papalillo, Andrea Martocchia \\ Department of Electronic Engineering, Università Degli Studi “Roma Tre”, Roma, Italy \\ Email: schirrip@uniroma3.it
}

Received October 12, 2011; revised November 17, 2011; accepted November 27, 2011

\begin{abstract}
In recent years, light emitting diodes (LEDs) have entered the lighting market, offering consumers performance and features exceeding those of traditional lighting technologies. LEDs (light-emitting diodes) are becoming more common in safety signals for railroad, highway, automotive, and many other applications. In addition to having a longer life and greater durability than incandescent bulbs, LEDs are much more energy efficient than their incandescent counterparts. Since the heat from the junction must be dissipated into the ambient somehow, changing the ambient temperature affects the junction temperature and hence the emitted light. When the LEDs are used in the railway or traffic signals, the optical proprieties of these have to maintain more rigorous specifications. Therefore the development of signals using LED as light source, able to respect intensity specifications, is not simple. In this paper, we describe problems of the temperature dependent changes of LED intensity and color shift. Besides we will introduce an innovative technique, that we have developed, to allow the use of the LEDs in applications with rigorous specifications.
\end{abstract}

Keywords: LED Temperature-Dependency; LED Intensity Shift; Railway Signal

\section{Introduction}

LEDs have been around since the 1960s, but they reach significant levels of luminous output only recently. Power, energy efficiency, maintenance savings, impact resistance durability make LEDs suitable for safety signals for railway, road traffic, and other applications in stationary transportation [1,2].

The new systems of signaling go to replace the conventional systems because they improve characteristics as the maintenance and the time of failure.

Conventional signals produce their lit aspects using either of following processes:

- tungsten lamps which emit nominally yellowish-white light and different combinations of colored and/or clear focusing lenses, which collect some of the light from the lamp, filter it into the appropriate color and focus it into the required angle.

- tungsten halogen lamps which emit nominally white light and with colored filters which develop the required color.

The major drawbacks of this technology are:

- limited duration of the light sources (about six months),

- low efficiency of conversion by electric input power to optical power,

- high risk of failure of signaling.
In particular, traditional light source fails catastrophically. Therefore, to guarantee the suitable safety standards, frequent interventions of maintenance and control are necessary. The regular, and frequent, replacement of filament lamps is labor intensive which results in high maintenance costs. This can be particularly high for a railway signal which requires a minimum of two staff (for safety) and which may be located at some distance from an accessible point along the railway. The reasons behind the replacement of filament lamps on the railway are thus reduction in maintenance costs. The replacement of filament lamp road traffic signals also provides reduction in maintenance and improved readability, but the main reason for utilizing LEDs is the reduction in power consumption and the consequent saving of energy $[3,4]$.

In any case the use of traditional lamps has the advantage that the intensity and chromaticity of emitted radiation appear to be stable and not influenced by changes in temperature. In contrast, the LED's technology available today has the defect that the intensity and dominant wavelength are significantly temperature dependent.

Thermal management is one of the major issues to be improved for implementing LEDs into lighting fixtures because heat affects the performance and reliability of those fixtures and LEDs. The optical proprieties of LEDs depended on junction temperature $[5,6]$. So that the new systems of signaling, using LEDs as light source, present 
new critical problems in order to respect the limits imposed by the norms. For signals used in stationary transportation, it is necessary to respect the prescriptions of project on ample range of temperature (typically 248 $348 \mathrm{~K})[7,8]$. The former consequence of temperature variation affects the output spectrum of the light, latter consequence is related to the intensity of radiation which decrease as the temperature increase.

The main topic of this research project is to determine a way to use the commercial available LEDs as light source in railway signals (as example we have considered the Italian railway signals), so that they respect the norms in each ambient temperature. To obtaining this goal, we study the thermal behavior of the LEDs used in those signals, like intensity and chromaticity. Then we study how to drive the chromaticity of the signal through intensity, in order to comply with the norms. At the end we propose the circuit solution able to drive the LED's intensity in the right way.

\section{Signal Characterization}

The railway signal under exam (as shown in Figure 1) is realized with some clusters of High-Power-LEDs of different type and color.

The clusters of LEDs are polarized with a constant current that it is invariant to changes of temperature. In order to verify the compliance of the norm at changing of ambient temperature of the railway signal provided, we implemented this test in a thermal chamber (see Figure 2). We change the ambient temperature while measuring the optical proprieties of the signal, for the three different color aspects.

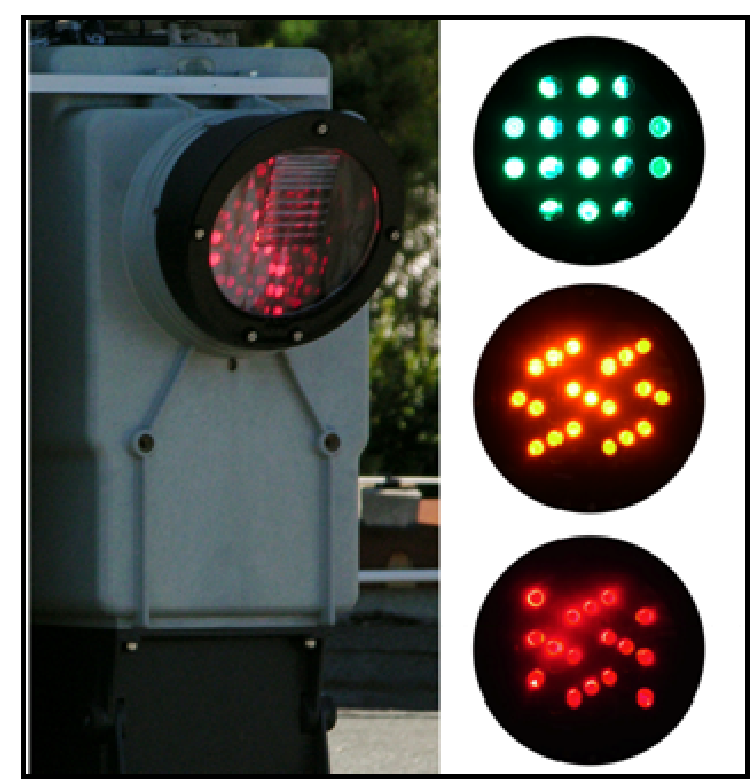

Figure 1. Typical Italian railway signal and example of LED matrix for different color aspects.
For what concerning the chromatic properties, we have used spectroradiometric measurement techniques through which we found the chromaticity coordinates of signals, according to the International Commission on Illumination (CIE). Our experience, about the three color aspects, pointed out that the yellow one has the most critical behavior. Using yellow LEDs realized in AlInGaP technology, it is difficult to achieve at extreme ambient temperature because the chromatic shift is acceptable only in a relative smaller range (263 - $323 \mathrm{~K}$ ) instead of the one specified by the norm (a typical example is shows in Figure 3).

From the previous spectroradimetric measurement, it was also possible to extract intensity performance of the three color aspects, which are represented in Figure 4. In the picture, the intensity curves are shown in straight line, while norm limits are in dash line. It is easy to point out, how the yellow aspect is the only one getting out of the norm for both low and high temperature limits.

These experiences prove that the main problem involving this railway signal is related to the compliance of the yellow aspect to Italian norm, which requires a strict chromatic condition, nearly monochromatic.

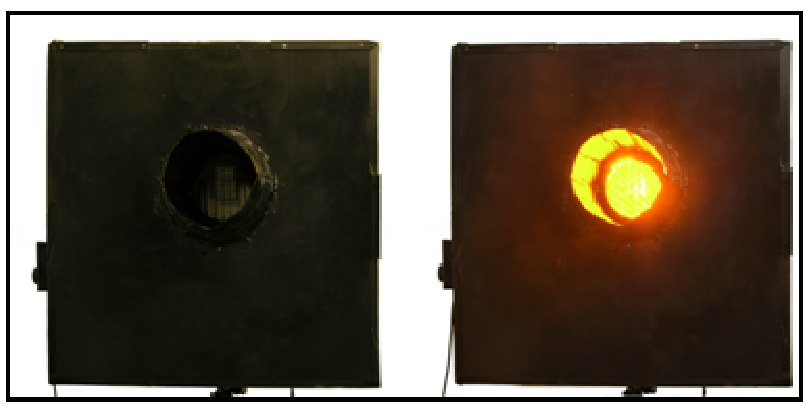

Figure 2. Thermal chamber built to test railway signals.

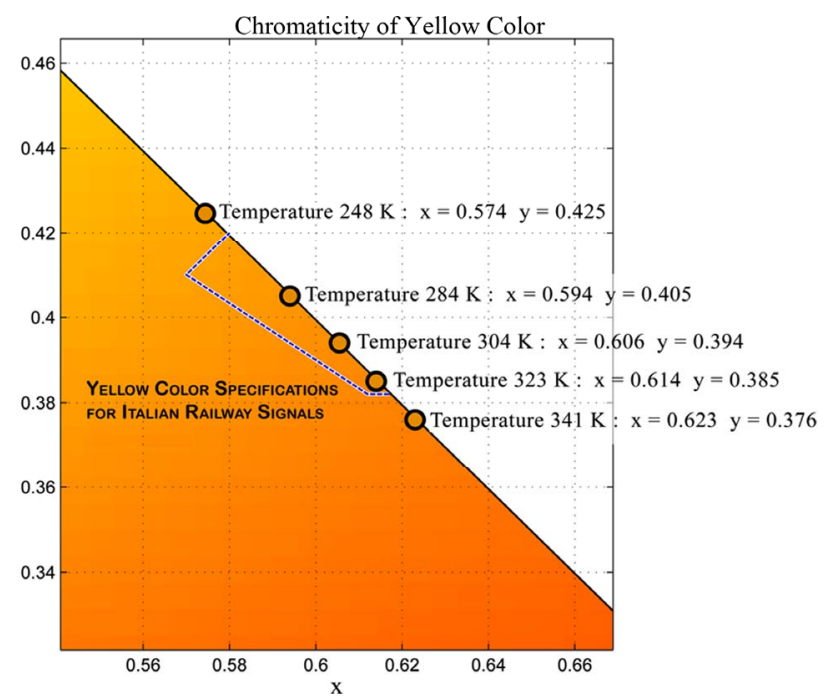

Figure 3. Colour stability problems associated with yellow AlInGaP LEDs. 


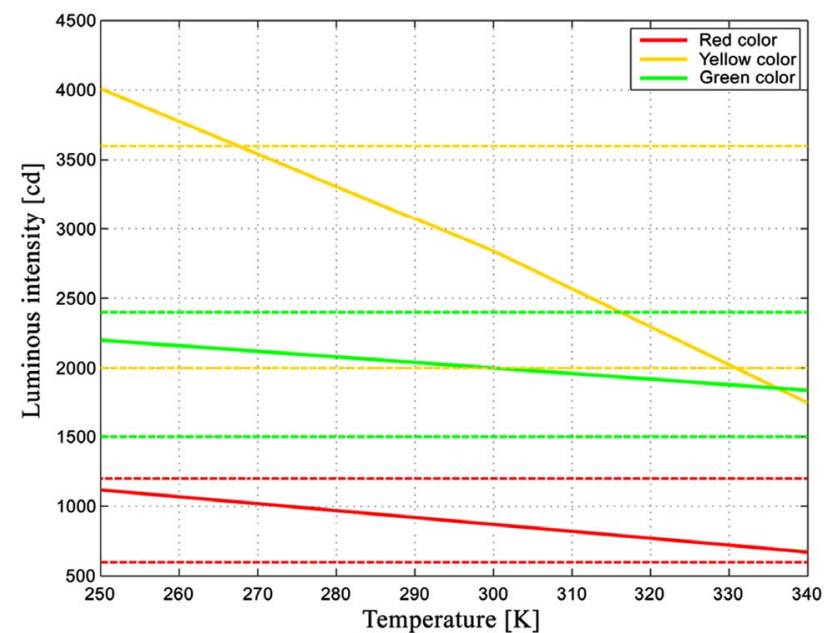

Figure 4. Intensity diagram for the yellow aspect as a function of ambient temperature.

Having a look to nowadays high-power LED's technology, it's simple to find out that GaN LEDs are principally used as small wavelength sources (UV, violet, blue, green), offering an inherent higher efficiency than the AlInGaP technology, which is still implied to provide wavelengths in the opposite side of the spectrum (see Figure 5).

Anyway, since GaN technology seems the flawless solution regarding the intensity performance of the LED source, this is not true anymore if compared to the emitting spectrum for the yellow aspect (see Figure 6) [9-11].

AlInGaP provides a narrower spectrum able to accomplish with the Italian norm, and it still remains the main technology used by the railway signals manufacturers for the yellow aspect.

For this reason, all our efforts to have an acceptable quality of the signal's illumination, are applied to stabilize the intensity of the AlInGaP-LED, as well as the thermaldrift of the chromaticity outside the norm's range.

\subsection{Chromatic Compensation}

For the chromatic aspect, our solution rely on a couple of LEDs similar to the ones used by the railway signals, which have different dominant wavelenghts:

- Osram LED LY E6SF-594 nm (yellow);

- AlInGaP technology;

- Osram LED LO E6SF-605 nm (orange);

- AlInGaP technology.

The purpose is to merge the two spectrum in order to obtain the suitable chromatic-behavior as the temperature becomes critical.

Then, we have built measurement setup similar to the one proposed by A. Groh, S. Brückner and T. Khanh [12], which allow us to set the thermal control over the junction of the LED. At the beginning of the measurement the LEDs packages are soldered onto the metal core board.

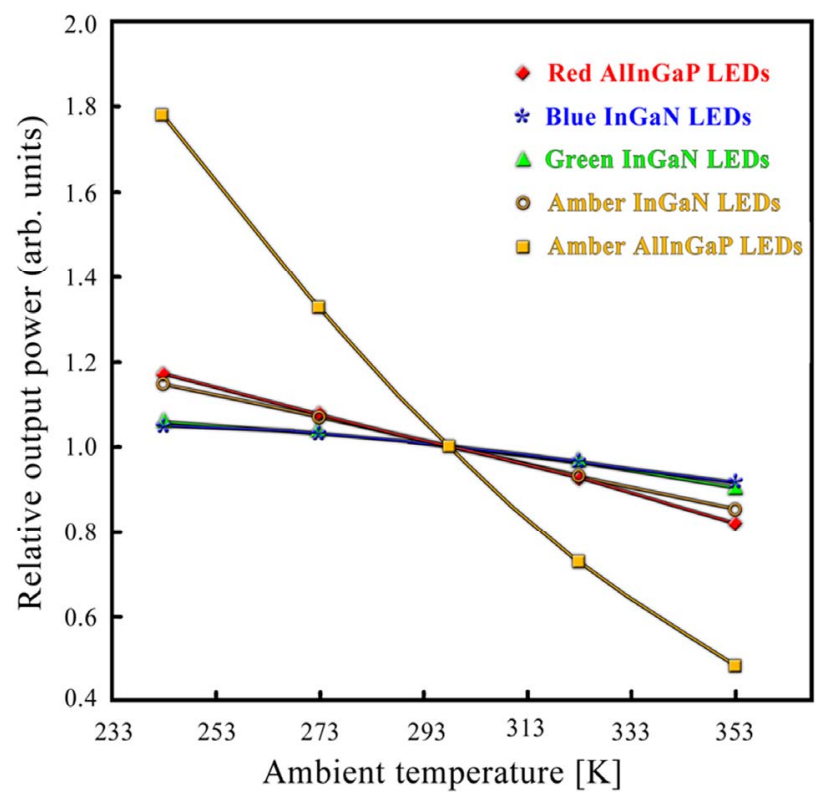

Figure 5. Output power of blue/green/amber InGaN and amber/red AlInGaP LEDs as a function of the ambient temperature from $233 \mathrm{~K}\left(-30^{\circ} \mathrm{C}\right.$ to $353\left(+80^{\circ} \mathrm{C}\right)$. The output power of each LED was normalized to 1.0 at $25^{\circ} \mathrm{C}$ [9].

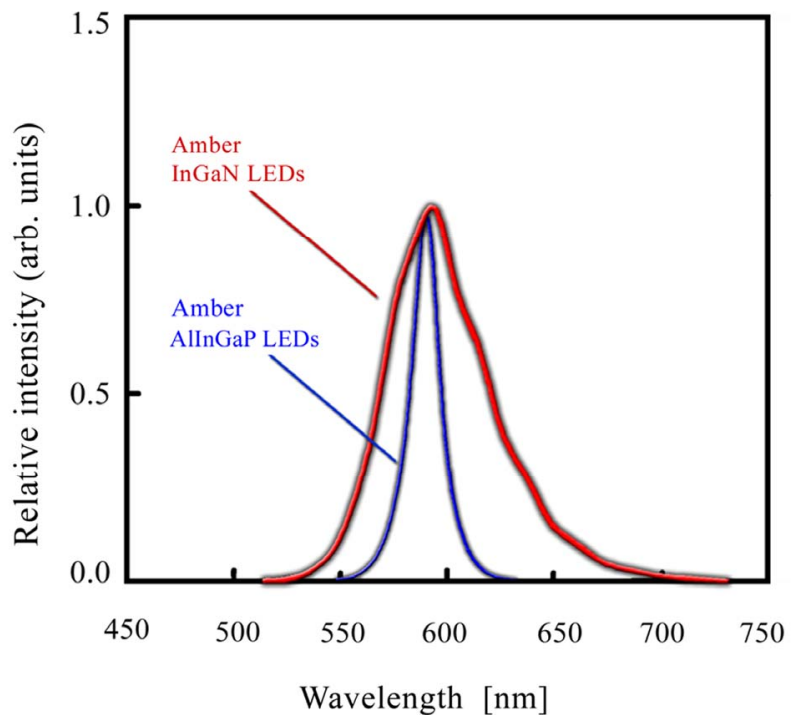

Figure 6. Emission spectra of amber InGaN, amber AlIn$\mathrm{GaP}$ at a forward current of $20 \mathrm{~mA}$ [9].

It is important that the solder pad of the LEDs packages exactly fit the solder pad of the metal core board in order to reach the lowest thermal resistance between LEDs and board. In this way, the junction temperature is nearly the same as the surface temperature of the die, being the $p n$-junction temperature the cause of shift of dominant wavelength. Afterwards the combination of the LEDs and the board is mounted on the temperature-controlled appliance. An outline of the whole structure is shown in Figure 7. 


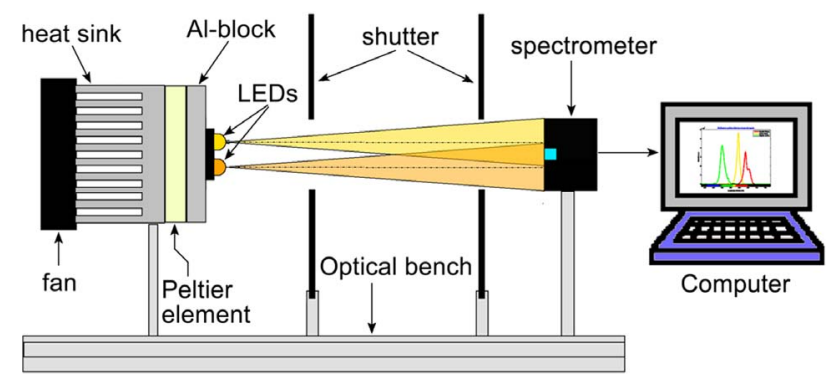

Figure 7. Outline of the measurement system.

The next step is to apply the needed current to the LEDs and wait until the structure is thermally stabilized. Since we have registered a higher junction temperature during the thermal characterization of the signal, we measured the spectrums of the LEDs, in a different temperature range from the one defined by the norm. The temperature of the substrate is controlled by a PID-controller combined with the Peltier element. The spectral power distribution is measured from $380 \mathrm{~nm}$ up to 780 $\mathrm{nm}$ with the spectrometer. All photometrical and colorimetrical parameters are calculated by software.

With this setup we have thermally characterized the spectrum of the LEDs in the same configuration of polarization, used by the railway signal. The measurement allows us to extract the chromatic coordinates of both LEDs in some significant temperature.

Now, we consider a new railway signal made up by a matrix of these two types of LED, so that its chromatic behavior is represented by the combination of each couple of chromatic coordinates (yellow and orange). Then, we use a software to manipulate the intensity ratio between the two types of LED, in order to obtain a resulting spectrum inside the norm limits, for every temperature conditions. So, if it is possible to define two electric powers for each type of LEDs, we can obtain a suitable optical characteristic for a signal, by mixing the chromatic coordinates weighted by the intensity of each LEDs. Also, if these electric powers piloted through measuring of temperature we could obtain a right behavior for all range of temperature, defined by the specifications (see Figures 8-10).

\subsection{Intensity Compensation}

Generally, the datasheets supply information around the electric/thermal behavior of the LED, and its optoelectronic properties, such as spectrum and intensity stability through thermal variations (see Figure 11) [13]. It is possible to see the dependence of forward current vs forward voltage at constant ambient temperature and forward voltage vs ambient temperature at constant current.

As we said before, an increasing temperature defines a drop in intensity performance, as well as a diminishing in the forward voltage. By measuring the forward voltage

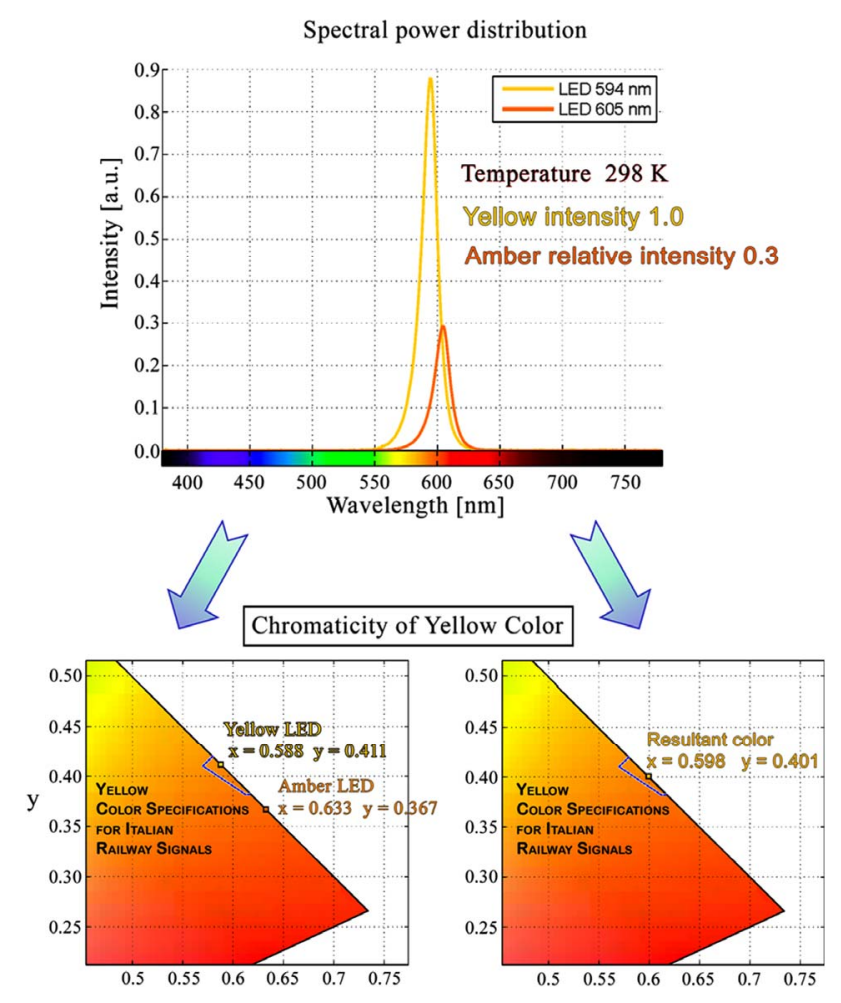

Figure 8. It shows that using the amber LED with intensity 0.3 times the intensity of the yellow LED, working at temperature of $298 \mathrm{~K}$, the resultant color falls at the center of color coordinates.
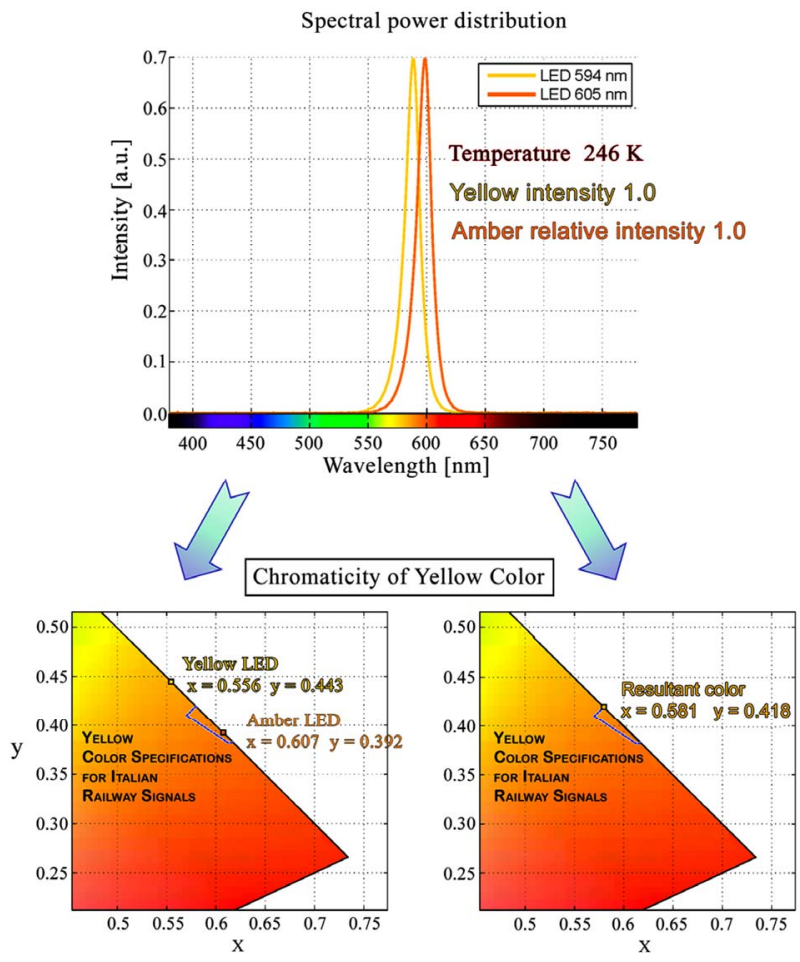

Figure 9. Working temperature $246 \mathrm{~K}$; amber LED with intensity equal the intensity of the yellow LED, the resultant color falls inside the color coordinates. 


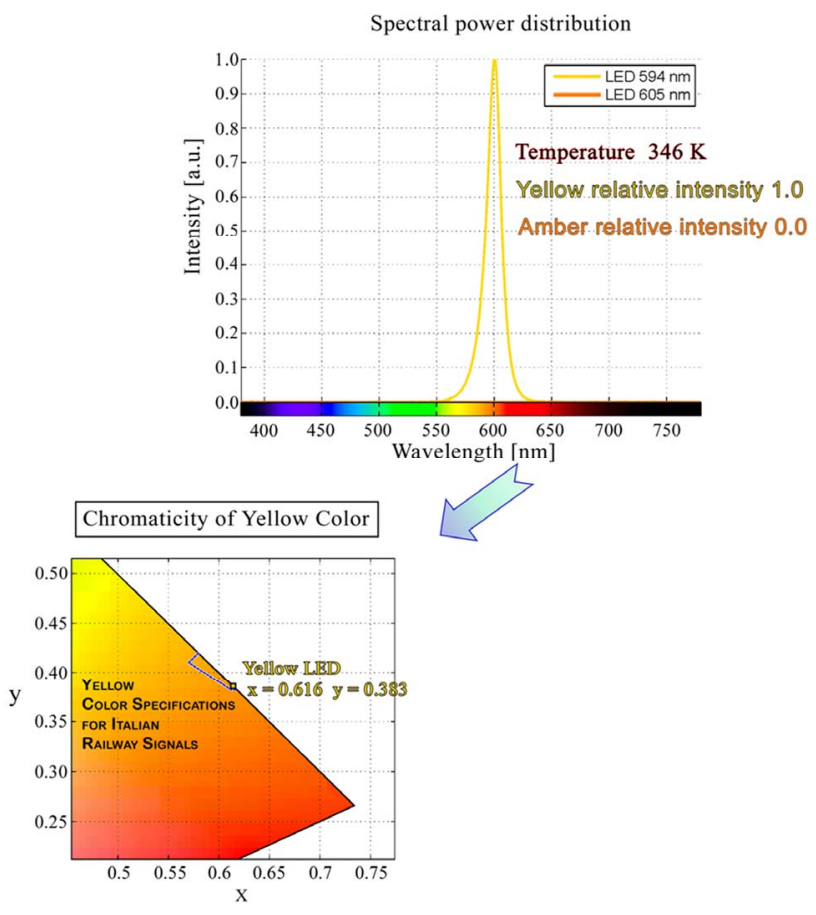

Figure 10. Working temperature $346 \mathrm{~K}$; amber LED is off, with this temperature yellow led have color inside the color coordinates.

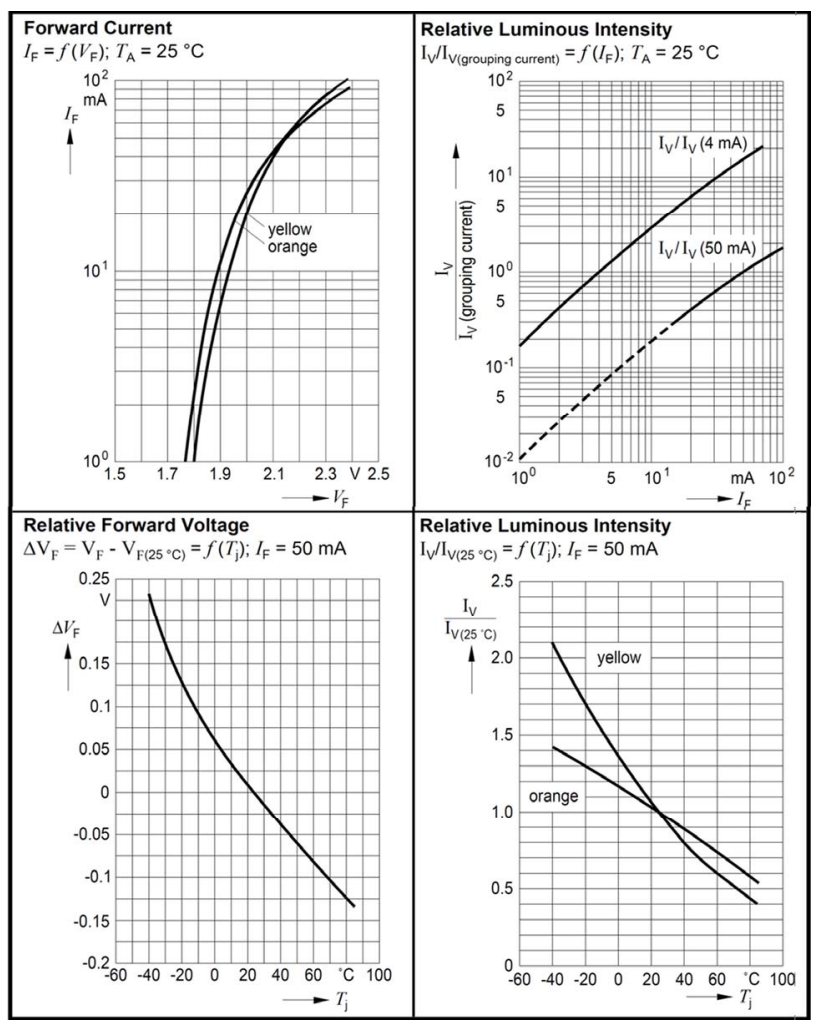

Figure 11. Characteristics of Osram LEDs; (a) Forward current vs Forward voltage; (b) Relative luminous intensity vs Forward current; (c) Relative forward voltage vs temperature; (d) Relative luminous intensity vs Temperature [13]. drop, it is possible to have a temperature reference of the LED cluster. This reference allows us to estimate the lowering in intensity, according to intensity-temperature relation described in the datasheet. It will be possible to compensate the intensity of radiation by increasing the forward current, since those parameters are related proportionally. However, if we increase the forward current, we lead the forward voltage to pursue this increment, so the compensation system is not trivial. Our solution provides a circuital feedback shown in Figure 12.

The core of the circuit is the red-marked op-amp, which works to maintain the equality of its inputs defining the polarization of the nMOS and so the current flowing in the cluster.

The circuit is designed to provide the following equation between the parameters of the LED-cluster:

$$
V_{F W}=-R_{S} I_{F W}+V_{R E F}
$$

The first instrumentation amplifier measures the forward voltage drop of the LED-cluster, due to thermaleffect, while the second amplifier takes the $R_{S} I_{F W}$. The red op-amp constrains the LED-cluster to work according to the relation (1), by increasing/decreasing the current only when the temperature raise/drop. The parameters of the circuit $R_{S}$ and $V_{R E F}$ define the work curve of the cluster (see Figure 13), and must tune the electrical behavior (voltage and current) to guarantee the thermal steadiness of the intensity. Looking at the curve, it is possible to understand the behavior of the circuit: as the temperature rises, the voltage drops, and the operating point (Q) drifts toward higher current, balancing the intensity loss.

Tuning these parameters lead us to merely compensate the thermal intensity drop, or to obtain a new intensity behavior for each temperature.

\section{Conclusions}

The ultimate goal of this study aims to design a new railway signaling system that combines the two proposed solutions for color stability and intensity. We have seen

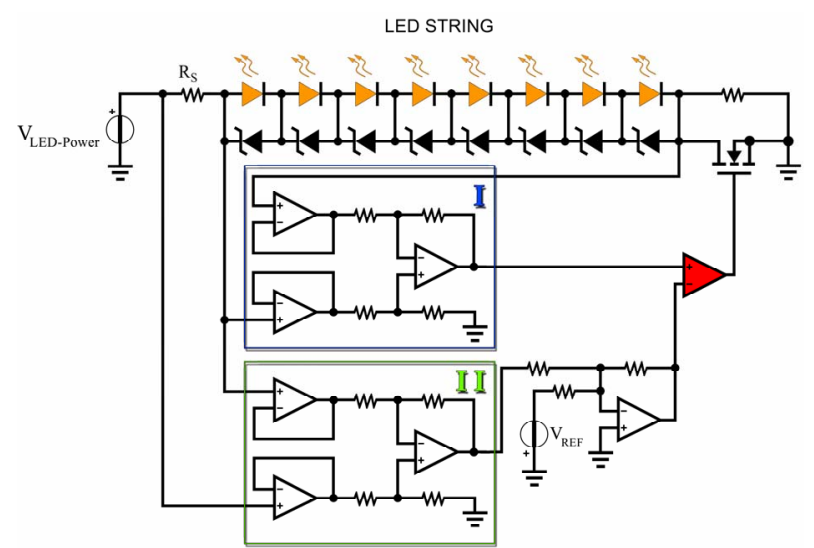

Figure 12. Circuital Setup for the intensity stability. 


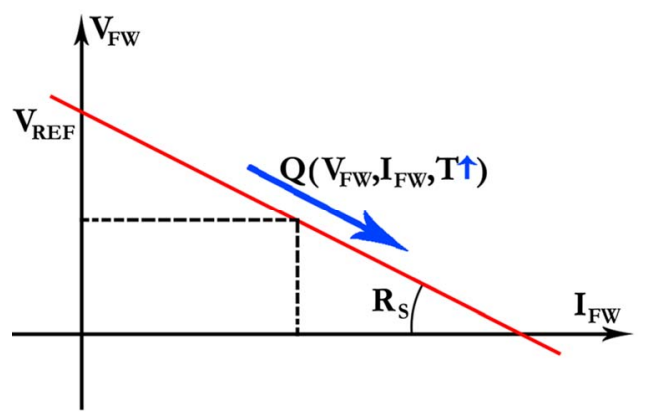

Figure 13. Curve of the Operating Point (Q) defined by circuit.

that using a pair of yellow and orange LEDs, it is possible to adjust the chromaticity in the temperature range indicated by the standard, only acting on their intensity ratio. For what concern the stability of intensity, we have seen a circuital setup able to counteract the drop in luminous efficiency with increasing temperature. The interesting aspect of the proposed circuit is related to the calibration of its parameters $\left(R_{S}\right.$ and $\left.V_{R E F}\right)$, which are able to provide any trend of intensity-temperature for a cluster of LED.

Assuming a signal consisting of a matrix of yellow and orange LED types, it will be possible to use and calibrate a couple of circuits for these two clusters of colors. As we said, this configuration allows to secure the thermalstability of intensity, but also the intensity trend to follow a predetermined temperature pattern. In this way it is possible to feed the clusters independently at any temperature, according to our design, and to obtain the optimal intensity ratio for the compliance of chromaticity over the whole range of temperatures.

\section{REFERENCES}

[1] W. Nobuo, “Recent LED Display Devices,” Japanese Railway Engineering, Vol. 119, 1992, pp. 6-8.

[2] E. F. Schubert, "Light-Emitting Diodes," Cambridge University Press, Cambridge, 2003.
[3] E. Hong and N. Narendran, "A Method for Projecting Useful Life of LED Lighting Systems,” 3rd International Conference on Solid State Lighting, Vol. 5187, 2004, pp. 93-99. doi:10.1117/12.509682

[4] N. Zheludev, "The Life and Times of the LED-A 100Year History,” Nature Photonics, Vol. 1, No. 4, 2007, pp. 189-192. doi:10.1038/nphoton.2007.34

[5] GmbH, "LED Metrology Handbook of LED Metrology," Instrument Systems GmbH, München, 2000, pp. 1-40.

[6] D. G. Todorov and L. G. Kapisazov, "LED Thermal Management,” ELECTRONICS’ 2008, Sozopol, 24-26 September 2008, pp. 139-144.

[7] ITE, "Vehicle Traffic Control Signal Heads (VTCSH)" and "VTCSH Part 2: LED Vehicle Signal Modules," Institute of Transportation Engineers, Washington DC.

[8] CIE, "A Guide for the Design of Road Traffic Lights," Commission International De L’Éclairage, Vienna, 1988.

[9] T. Mukai, M. Yamada and S. Nakamura, "Characteristics of InGaN-Based UV/Blue/Green/Amber/Red Light-Emitting Diodes,” Japanese Journal of Applied Physics, Vol. 38, No. 7A, 1999, pp. 3976-3981. doi:10.1143/JJAP.38.3976

[10] F. A. Kish and R. M. Fletcher, "AlInGaP Light-Emitting Diodes,” In: G. B. Stringfellow and M. G. Crawford, Eds., Semiconductors and Semimetals, Academic Press, San Diego, 1997, p. 149.

[11] T. Suski, P. Perlin and B. Monemar, "Gallium Nitride (GaN) I,” In: J. I. Pankove and T. D. Moustakas, Eds., Semicondutors and Semimetals, Academic Press, San Diego, 1998, p. 279.

[12] A. Groh, S. Brückner and T. Q. Khanh, "The Temperature-Dependent Changes of the Photometrical and Colorimetrical Parameters of Today High Power LEDs,” Proceedings of the 7th International Symposium on Automotive Lighting, Munchen, 25-26 September 2007, pp. 622-629.

[13] OptoSemicondutors OSRAM, LS E6SF, LR E6SF, LA E6SF, LO E6SF, LY E6SF, datasheet.

http://catalog.osram-os.com/catalogue/catalogue.do?act=d ownloadFile\&favOid=0200000200008f8a000200b6 